\title{
Variabilidad del peso, la estatura y el índice de masa corporal según desarrollo puberal y tipo de colegio en adolescentes de Medellín, Colombia
}

\author{
Weight, height and body mass index variability according \\ to both puberal development and to the type of \\ school in adolescents of Medellín, Colombia
}

\begin{abstract}
Introduction: Studies of growth variation in adolescents according to puberal development and socio-economic conditions is lacking for Medellin city. Aim: to analyze weight, height and body mass index (BMI) variability according to puberal development and type of school in adolescents from Medellin. Subjects and methods: Adolescents of both sexes at stages II to V of Tanner puberal development previously involved in the study "Cardiovascular risk factors in Medellin 1998-1999" were selected. Weight, height and BMI (Kg/m2) data were analyzed according to puberal development and type of school (public or private). Results: Males from private schools had weight $(p=0.000)$, height $(p=0.001)$ and $B M I(p=0.002)$ greater than those from public schools, without interaction of puberal development and type of school ( $p>0.05)$. In females, weight and height were greater in adolescents from private schools $(p=0.000)$ showing an interaction between $p u$ beral development and type of school (weight $p=0.013$; height $p=0.040)$; school type did not influence BMI ( $p=0.347)$, although there was interaction with puberal development $(p=0.013)$. In the beginning $B M I$ was greater for private schools women and then the situation became inverted at the end of puberal development (Tanner V). Conclusion: puberal development influences both males and females growth. However, the different magnitude on $B M I$ and stages at which they occur could be related to cultural factors in women.
\end{abstract}

Key words: adolescent, body weight, height, body mass index, puberal development, type of school.

\section{INTRODUCCIÓN}

En la adolescencia se presentan grandes cambios físicos influenciados por el grado de desarrollo puberal, que se refiere a un complejo proceso de crecimiento conocido como pubertad. Este proceso se caracteriza, entre otros aspectos, por la aparición de los caracteres sexuales secundarios, la aceleración del crecimiento en estatura y peso $(1,2)$. Varios autores han documentado la relación entre el desarrollo puberal y el crecimiento físico; al respecto, Mauricci y col. observaron que la estatura final alcanzada por adolescentes chilenos difería según la edad de inicio de la pubertad (3).

En una muestra de adolescentes chilenos, recolectada entre 1985 y 1988 (4), y en otra de varones adolescentes venezolanos en 2007 (5), se encontró que aquellos con la misma
Argenis Giraldo G. (1)

Rosa Magdalena Uscátegui P. (1)

María Teresa Restrepo C. (1)

Alejandro Estrada R. (2)

1) Grupo de Investigación en Alimentación y Nutrición Humana, Escuela de Nutrición y Dietética, Universidad de Antioquia, Medellín, Colombia. (2) Grupo de Demografía y Salud, Facultad Nacional de Salud Pública, Universidad de Antioquia, Medellín, Colombia.

Dirigir la correspondencia a: Profesora

Argenis Giraldo García Laboratorio de Antropometría Escuela de Nutrición y Dietética Universidad de Antioquia Cra. 75 N $65-87$, bloque 44 of. 204, Medellín, Colombia Teléfono: (57-4)- 2199232 Fax: (57-4) 2305007

E-mail:argenisgiraldo@gmail.com

Este trabajo fue recibido el 25 de Enero de 2012 y aceptado para ser publicado el 8 de Agosto de 2012. edad y distinto estadio de desarrollo puberal presentaban diferencias de IMC, por el contrario cuando el análisis se hizo en sujetos de igual estadio de desarrollo puberal y distinta edad, las diferencias no fueron significativas.

El crecimiento durante la pubertad depende de la interacción de factores genéticos con ambientales, entre estos últimos destaca la condición socioeconómica (6). Los adolescentes que viven en mejores condiciones de vida tienen mayor peso y estatura y presentan un desarrollo puberal a edades más tempranas, comparadas con adolescentes de la misma región en condiciones menos favorables $(7,8)$.

Algunos estudios en población escolarizada usaron la clasificación por tipo de colegio público y privado como indicador de las condiciones socioeconómicas, relacionadas con el gasto 
en educación, puesto que los colegios públicos son subvencionados parcial o totalmente por el Estado, mientras que los privados implican gastos en matrículasy mensualidades (9-11).

En un estudio realizado en Medellín, en 1983, en hombres y mujeres de 6 a 18 años de edad, se observaron promedios de estatura superiores en los escolares de los colegios privados frente a aquellos de los públicos, pero no se consideró el grado de desarrollo puberal (11). Igualmente, otra investigación desarrollada en Medellín, en 1998, en adolescentes de 14 a 17 años, mostró que los pospúberes del estrato alto presentaron los mayores promedios de estatura (12); sin embargo este estudio no señala la magnitud de las diferencias en peso, estatura e IMC según desarrollo puberal, por el corto rango de edad estudiado (14-17 años). En Medellín, no se cuenta con información sobre el efecto combinado del desarrollo puberal y las condiciones socioeconómicas sobre la variabilidad del peso, la estatura y el IMC, por lo tanto, este estudio es un punto de partida que permitirá conocer cómo se presentan los cambios físicos en relación con factores ontogénicos y socioeconómicos. El objetivo de este estudio fue analizar la variabilidad del peso, la estatura y el IMC según desarrollo puberal y tipo de colegio en adolescentes escolarizados de Medellín.

\section{SUJETOS Y MÉTODO}

Se realizó un estudio descriptivo de corte transversal, en el cual se utilizó la muestra de la investigación "Prevalencia de algunos factores de riesgo cardiovascular, en población escolarizada de primaria y secundaria, de la jornada diurna de los colegios de Medellín, Colombia", realizada por un grupo interdisciplinario de la Universidad de Antioquia, entre 1998 y 1999 (13). La base de datos se utilizó con autorización del investigador principal del estudio en mención. En dicha investigación, la muestra se calculó de acuerdo a los siguientes parámetros: un nivel de confianza de 95\%, un error de muestreo de $2 \%$ y una probabilidad de ocurrencia de 50\%; quedó conformada por 2620 sujetos. Esta muestra se convierte en la fuente de datos secundaria para el presente estudio. Del total de la misma se descartaron los niños en estadio I de desarrollo puberal, quedando para el análisis 1305 adolescentes.

Mediciones antropométricas: se realizaron en todos los sujetos por el mismo evaluador, siguiendo las técnicas unificadas internacionalmente por un grupo de expertos (14). El peso se midió en kilogramos con una balanza electrónica marca Detecto ${ }^{\circledR}$, de $200 \mathrm{~kg}$ de capacidad y 0,1 kg de sensibilidad y la talla en centímetros con un estadiómetro marca Jandac , de $220 \mathrm{~cm}$ de longitud y 0,1 cm de precisión. Cada medida se evaluó y registró dos veces, y el promedio se tomó como dato definitivo. Si la diferencia en talla era mayor de $0,5 \mathrm{~cm}$ y en peso de 0,1 kg, las mediciones se tomaron una tercera vez. El IMC de cada participante se obtuvo mediante la división del peso en $\mathrm{kg}$ por la estatura en $\mathrm{m} 2(\mathrm{~kg} / \mathrm{m} 2)$. Para el análisis del peso, la talla y el IMC se usaron los valores absolutos (13).

Evaluación del desarrollo puberal: la realizó personal médico con experiencia en este tipo de valoración, mediante examen físico, siguiendo la metodología propuesta por Tanner $(15,16)$, la cual establece cinco estadios de maduración según el desarrollo de genitales externos en los hombres y el desarrollo mamario en las mujeres.

Condiciones socioeconómicas: se midieron tomando como indicador el tipo de colegio, público o privado, donde estudiaba el adolescente; este indicador constituye una medida indirecta del gasto en educación, puesto que los colegios públicos en Medellín son gratuitos, mientras que los privados requieren el pago de matrícula y mensualidades.

\section{ANÁLISIS ESTADÍSTICO}

Inicialmente se realizó estadística descriptiva para caracterizar la población. La evaluación del efecto del desarrollo puberal, el tipo de colegio y la interacción entre ellos, sobre el peso, la estatura y el IMC, se realizó por análisis de varianza (ANOVA) de dos factores, previa transformación del peso y el IMC, utilizando el método de potencia Box-Cox; el peso en las mujeres se normalizó mediante la raíz cuadrada y en los hombres por medio de valores predichos, obtenidos mediante un modelo de regresión lineal múltiple, cuya variable dependiente fue el peso transformado según su inverso cuadrático. El IMC en las mujeres se transformó mediante el inverso de la raíz cuadrada y en los hombres mediante su inverso cuadrático. Puesto que la edad cronológica es una variable interviniente, se incluyó como covariable en el ANOVA de dos factores para controlar su efecto, cuando se encontró efecto positivo del desarrollo puberal, se realizó la prueba de comparación múltiple de Bonferroni.

Se probó el supuesto de normalidad mediante la prueba de Kolmogorov-Smirnov y el de homoceasticidad por medio de la prueba de Levene para los diferentes modelos.

El análisis se efectuó en el SPSS $®$ versión 17 (Statistical Package for the Social Sciences). Se consideraron diferencias significativas cuando los valores de p fueron inferiores a 0,05.

Aspectos éticos de la investigación: el presente estudio contó con el aval del Comité de Ética de la Sede de Investigación Universitaria de la Universidad de Antioquia. La investigación "Prevalencia de algunos factores de riesgo cardiovascular, en población escolarizada de primaria y secundaria, de la jornada diurna de los colegios de Medellín, Colombia", de la que se obtuvo la base de datos, tenía aprobación del Comité de Ética de la Facultad de Medicina de la misma universidad, y contaba con el consentimiento informado firmado por los padres o responsables del adolescente.

\section{RESULTADOS}

Se estudiaron 1305 adolescentes, 36,6\% hombres (477) y $63,4 \%$ mujeres (828), de los cuales $66,6 \%$ (869) estudiaban en colegios públicos y $33,4 \%$ (436) en colegios privados. Los adolescentes evaluados estaban entre los estadios II y $\mathrm{V}$ de desarrollo puberal según la clasificación de Tanner; la muestra se describe en el tabla 1.

\section{Efecto del desarrollo puberal y tipo de colegio} sobre los datos antropométricos

En los varones, la edad tuvo efecto significativo en el peso y la estatura, pero no en el IMC (tabla 2). El peso aumentó a medida que se incrementaba el grado de desarrollo puberal $(p=0,000)$, en la mayoría de los casos la comparación del peso entre parejas de estadios de desarrollo puberal fue significativamente diferente $(p<0,05)$ (tabla 3$)$. Igualmente, el tipo de colegio tuvo un efecto significativo sobre el peso $(p=0,000)$, con valores más altos en los varones de los colegios privados, pero no se presentó interacción entre el desarrollo puberal y el tipo de colegio ( $p=0,261)$ (figura 1a).

La estatura en los varones varió según el grado de desarrollo puberal $(p=0,000)$, las diferencias entre los distintos estadios fueron significativas, con excepción de la comparación entre los estadios IV y V (tabla 3). De igual forma, el tipo de colegio tuvo efecto significativo sobre la estatura $(p=0,001)$, con valores superiores en los hombres de los colegios privados y sin interacción entre el tipo de colegio y el desarrollo puberal $(p=0,235)$; es decir, al interior de cada estadio de desarrollo puberal la estatura por tipo de colegio mostró el mismo com- 
portamiento (tabla 2).

La prueba ANOVA reveló el efecto del desarrollo puberal sobre el IMC en los hombres ( $p=0,000)$; este fue mayor en los estadios sexuales más altos; sin embargo, la comparación por pares de estadios de desarrollo de genitales externos no reveló diferencias significativas entre los estadios II y III, III y IV de los varones de los colegios públicos y los estadios II y III, II y IV, III y IV y IV y V de los colegios privados (tabla 3). El tipo de colegio también tuvo efecto sobre el IMC ( $p=0,001)$, los valores fueron mayores en los hombres de los colegios privados y no se observó interacción entre el grado de desarrollo puberal y el tipo de colegio (tabla 2).

En las mujeres se encontró que la edad tuvo efecto significativo en el peso, la estatura y el IMC. Por su parte, tanto el desarrollo puberal $(p=0,000)$ como el tipo de colegio $(p=0,000)$, y la interacción de ambos factores $(p=0,013)$, tuvieron un efecto sobre el peso (tabla 2 y figura $1 \mathrm{~d}$ ). Los valores fueron más altos en las mujeres de colegios privados y la mayor diferencia del peso según el tipo de colegio se observó en el estadio III. El test de comparaciones múltiples reveló que las diferencias de peso entre los estadios de desarrollo puberal fueron significativas en casi todas las comparaciones $(p<0,05)$, a excepción de la comparación de los estadios III y IV de las mujeres de los colegios privados (tabla 3 ). Igualmente, se encontró un efecto del desarrollo puberal sobre la estatura $(p=0,000)$, lo mismo que el tipo de colegio $(p=0,000)$ e interacción entre este y el desarrollo puberal $(p=0,040)$ (tabla 2 y figura 1e), con diferencias significativas de la estatura entre algunos de los estadios de desarrollo mamario (tabla 3). La estatura fue mayor en las mujeres de los colegios privados y la diferencia con los públicos se hizo mayor a medida que se incrementaba el grado de desarrollo puberal.

En el IMC de las mujeres, se encontró un efecto significativo del desarrollo puberal $(p=0,000)$, la comparación múltiple del IMC entre los distintos estadios de desarrollo mamario mostró diferencias significativas en la mayoría de los casos (tabla 3), el tipo de colegio no presentó efecto sobre el IMC $(p=0,347)$, pero sí se observó interacción entre el desarrollo puberal y el tipo de colegio $(p=0,013)$. De hecho, los valores de IMC en el estadio II de desarrollo puberal fueron mayores en las mujeres de los colegios privados, pero esta situación se invirtió en los estadios IV y V (tabla 3 y figura 1f).

TABLA 1

Descripción de la población analizada

\begin{tabular}{|c|c|c|c|c|c|c|c|c|c|}
\hline \multicolumn{6}{|c|}{ Hombres } & \multicolumn{4}{|c|}{ Mujeres } \\
\hline \multirow{3}{*}{$\begin{array}{l}\text { Grado } \\
\text { MP }\end{array}$} & \multicolumn{2}{|c|}{ Tipo de colegio } & \multirow{3}{*}{$\begin{array}{c}\text { Total } \\
\text { (años) } \\
n\end{array}$} & \multirow{3}{*}{$\begin{array}{l}\text { Edad } \\
\text { (años) } \\
X \pm D E\end{array}$} & \multirow{3}{*}{$\begin{array}{c}\text { Grado } \\
\text { MP }\end{array}$} & \multirow{2}{*}{\multicolumn{2}{|c|}{$\begin{array}{l}\text { Tipo de colegio } \\
\text { Público }\end{array}$}} & \multirow{3}{*}{$\begin{array}{c}\text { Total } \\
\text { Privado } \\
n\end{array}$} & \multirow{3}{*}{ 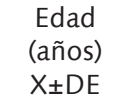 } \\
\hline & Público & Privado & & & & & & & \\
\hline & $\mathrm{n}$ & $\mathrm{n}$ & & & & $\mathrm{n}$ & $\mathrm{n}$ & & \\
\hline$G \|$ & 83 & 28 & 111 & $12,5 \pm 1,5$ & $M I I$ & 153 & 43 & 196 & $10,7 \pm 1,2$ \\
\hline G III & 60 & 19 & 79 & $14,2 \pm 1,5$ & $M$ III & 71 & 45 & 116 & $12,4 \pm 1,8$ \\
\hline G IV & 106 & 46 & 152 & $15,8 \pm 1,5$ & M IV & 191 & 119 & 310 & $14,9 \pm 2,0$ \\
\hline$G \vee$ & 73 & 62 & 135 & $17,2 \pm 1,2$ & $M V$ & 132 & 74 & 206 & $16,2 \pm 1,6$ \\
\hline
\end{tabular}

Grado MP: grado de desarrollo puberal, la G significa genital y la M mamario

$\mathrm{X} \pm \mathrm{DE}$ : Promedio \pm desviación estándar

\section{TABLA 2}

Efecto del desarrollo puberal, el tipo de colegio y la interacción de ambos sobre el peso, la estatura y el IMC en hombres y mujeres, ajustado por edad (ANOVA de dos factores)

\begin{tabular}{|c|c|c|c|c|c|c|c|}
\hline \multirow[t]{2}{*}{ Variable } & \multirow[t]{2}{*}{ Factor } & \multicolumn{3}{|c|}{ Hombres } & \multicolumn{3}{|c|}{ Mujeres } \\
\hline & & $g \mid$ & $\mathrm{F}$ & $\mathrm{p}$ & gl & $\mathrm{F}$ & $\mathrm{p}$ \\
\hline \multirow[t]{4}{*}{ Peso } & Desarrollo puberal & 3 & 36,28 & 0,000 & 3 & 61,07 & 0,000 \\
\hline & Tipo de colegio & 1 & 21,38 & 0,000 & 1 & 20,29 & 0,000 \\
\hline & Desarrollo puberal * tipo de colegio & 3 & 1,34 & 0,261 & 3 & 3,62 & 0,013 \\
\hline & Edad & 1 & 99,88 & 0,000 & 1 & 70,53 & 0,000 \\
\hline \multirow[t]{4}{*}{ Estatura } & Desarrollo puberal & 3 & 50,92 & 0,000 & 3 & 51,60 & 0,000 \\
\hline & Tipo de colegio & 1 & 12,08 & 0,001 & 1 & 51,48 & 0,000 \\
\hline & Desarrollo puberal * tipo de colegio & 3 & 1,42 & 0,235 & 3 & 2,79 & 0,040 \\
\hline & Edad & 1 & 101,88 & 0,000 & 1 & 87,11 & 0,000 \\
\hline \multirow[t]{4}{*}{ IMC } & Desarrollo puberal & 3 & 10,96 & 0,000 & 3 & 30,95 & 0,000 \\
\hline & Tipo de colegio & 1 & 10,05 & 0,002 & 1 & 0,88 & 0,347 \\
\hline & Desarrollo puberal * tipo de colegio & 3 & 0,65 & 0,586 & 3 & 3,61 & 0,013 \\
\hline & Edad & 1 & 1,25 & 0,264 & 1 & 13,10 & 0,000 \\
\hline
\end{tabular}




\section{DISCUSIÓN}

Los mayores pesos y estaturas asociados con las mejores condiciones socioeconómicas, que se observaron en la muestra de adolescentes de Medellín, están de acuerdo con los reportes de otros investigadores, que han documentado estaturas superiores en los de estratos más altos. Los sujetos que viven en condiciones socioeconomicas desfavorables son de menor estatura, de dimensiones corporales más pequeñas y con frecuencia presentan mayor déficit nutricional $(1,17)$. Si bien las condiciones socioeconómicas al nacimiento son determinantes en gran medida de la talla a los 19 años de edad, el empeoramiento de dichas condiciones entre el nacimiento y la adolescencia también altera la estatura alcanzada al finalizar el crecimiento; es el caso de individuos que no eran pobres en el momento del nacimiento, pero se empobrecieron durante la niñez o la adolescencia, quienes presentaron un promedio de estatura intermedio, entre aquellos que nunca fueron pobres y los que fueron pobres desde el momento del nacimiento, lo que podría estar relacionado con un crecimiento más lento durante el estirón (18).

Una de las investigaciones que consideró los cambios físicos por estadios de desarrollo puberal fue publicada en 2010 por Burrows y cols(19), quienes comparararon los datos de estatura e IMC según desarrollo puberal, en una muestra obtenida en el año 1986 y otra en 2001, de adolescentes chilenos de ambos sexos, pertenecientes a colegios subvencionados por el Estado de la Región Metropolitana de Chile, donde estudiaban niños pertenecientes al nivel socioeconómico medio. Se tomaron los datos de 2001 para compararlos con los del presente estudio por la proximidad de tiempo en que fueron obtenidos, puesto que la investigación de factores de riesgo cardiovascular de la cual se derivó la muestra de los niños de Medellín fue realizada entre 1998 y 1999.

La comparación de la estatura según desarrollo puberal de los varones de colegios públicos y privados de Medellín con relación a los de Chile reveló estaturas ligeramente más bajas en los hombres de la ciudad. En cambio, en las mujeres dicha comparación mostró que al final del desarrollo puberal (estadio V), las jóvenes de los colegios públicos de Medellín tuvieron $2,5 \mathrm{~cm}$ menos de estatura que las chilenas, mientras la estatura de aquellas adolescentes de los colegios privados fue $1,5 \mathrm{~cm}$ mayor que de las chilenas. Los datos en las niñas están de acuerdo con las condiciones socioeconómicas de los grupos comparados, puesto que las niñas chilenas que asisten a colegios subvencionados son de un estrato medio (19).

El IMC aumentó en ambos sexos según estadios de desarrollo puberal, situación que ha sido reportada en tres estudios latinoamericanos $(4,5,20)$. Según Guerrero, el aumento significativo y gradual del IMC en sujetos de similar edad cronológica al avanzar en cada etapa de desarrollo puberal expresa su relación con la edad biológica más que con la cronológica (5).

EI IMC es el indicador recomendado por la OMS para diagnosticar sobrepeso y obesidad, en 2007 se propusieron estándares de IMC para uso internacional (21), estratificados por edad cronológica, los cuales fueron aceptados para Colombia por el Ministerio de la Protección Social mediante resolución 2121 de 2010 (22); sin embargo se debe tener en cuenta la influencia del desarrollo puberal sobre el IMC en la evaluación

TABLA 3

Comparaciones múltiples de peso, estatura e IMC según desarrollo puberal y tipo de colegio, ajustados por edad

\begin{tabular}{|c|c|c|c|c|c|c|}
\hline Sexo & $\begin{array}{l}\text { Tipo de } \\
\text { colegio }\end{array}$ & $\begin{array}{l}\text { Grado } \\
\text { DP }\end{array}$ & $\mathrm{n}$ & $\begin{array}{l}\text { Peso* } \\
X \pm E E\end{array}$ & $\begin{array}{l}\text { Estatura (cm) } \\
\qquad X_{ \pm} \mathrm{EE}\end{array}$ & $\begin{array}{l}I M C \neq \\
X \pm E E\end{array}$ \\
\hline Hombres & Público & $\begin{array}{l}\text { G II } \\
\text { G III } \\
\text { G IV } \\
\text { G V } \\
p^{\| \prime} \\
\text { G II } \\
\text { G III } \\
\text { G IV } \\
\text { G V } \\
p^{\| I}\end{array}$ & $\begin{array}{c}83 \\
60 \\
105 \\
73 \\
\\
28 \\
19 \\
46 \\
62\end{array}$ & $\begin{array}{c}0,0003 \pm 0,00001^{\mathrm{a}} \\
0,0002 \pm 0,00001^{\mathrm{b}} \\
0,0002 \pm 0,00001^{\mathrm{c}} \\
0,0002 \pm 0,00001^{\mathrm{c}, \mathrm{d}} \\
0,000 \\
0,0002 \pm 0,00001^{\mathrm{a}} \\
0,0001 \pm 0,00001^{\mathrm{b}} \\
0,0001 \pm 0,00001^{\mathrm{b}, \mathrm{c}} \\
0,0001 \pm 0,00001^{\mathrm{b}, \mathrm{c}, \mathrm{d}} \\
0,000\end{array}$ & $\begin{array}{c}152,62 \pm 1,072^{\mathrm{a}} \\
158,21 \pm 0,948^{\mathrm{b}} \\
165,16 \pm 0,746^{\mathrm{c}} \\
164,63 \pm 1,048^{\mathrm{c}, \mathrm{d}} \\
0,000 \\
153,00 \pm 1,416^{\mathrm{a}} \\
161,95 \pm 1,336^{\mathrm{b}} \\
169,11 \pm 0,793^{\mathrm{c}} \\
167,27 \pm 0,915^{\mathrm{c}, \mathrm{d}} \\
0,000\end{array}$ & $\begin{array}{c}0,002 \pm 0,00006^{a} \\
0,002 \pm 0,00006^{a, b} \\
0,002 \pm 0,00004^{b, c} \\
0,001 \pm 0,00006^{d} \\
0,000 \\
0,002 \pm 0,00011^{a} \\
0,002 \pm 0,00010^{a, b} \\
0,002 \pm 0,00006^{a, b, c} \\
0,001 \pm 0,00007^{b, c, d} \\
0,023\end{array}$ \\
\hline Mujeres ${ }^{\dagger, \S}$ & Público & $\begin{array}{l}M \text { II } \\
M \text { III } \\
M \text { IV } \\
M \text { V } \\
p^{\| I} \\
M I I \\
M I I I \\
M I V \\
M V \\
p^{\| I}\end{array}$ & $\begin{array}{c}153 \\
71 \\
191 \\
132 \\
43 \\
45 \\
19 \\
74\end{array}$ & $\begin{array}{c}10,19 \pm 0,113^{\mathrm{a}} \\
10,68 \pm 0,127^{\mathrm{b}} \\
11,51 \pm 0,078^{\mathrm{c}} \\
12,17 \pm 0,113^{\mathrm{d}} \\
0,000 \\
10,42 \pm 0,172^{\mathrm{a}} \\
11,65 \pm 0,138^{\mathrm{b}} \\
11,83 \pm 0,086^{\mathrm{b}, \mathrm{c}} \\
12,53 \pm 0,112^{\mathrm{d}} \\
0,000\end{array}$ & $\begin{array}{c}142,59 \pm 0,734^{a} \\
147,99 \pm 0,829^{b} \\
152,29 \pm 0,510^{c} \\
152,38 \pm 0,737^{c, d} \\
0,000 \\
145,61 \pm 1,134^{a} \\
154,24 \pm 0,909^{b} \\
156,32 \pm 0,565^{b, c} \\
157,52 \pm 0,743^{c, d} \\
0,000\end{array}$ & $\begin{array}{c}1,53 \pm 0,003^{\mathrm{a}} \\
1,53 \pm 0,004 \mathrm{a}^{\mathrm{b}} \\
1,55 \pm 0,002^{\mathrm{c}} \\
1,57 \pm 0,003^{\mathrm{d}} \\
0,000 \\
1,53 \pm 0,004^{\mathrm{a}} \\
1,55 \pm 0,004^{\mathrm{b}} \\
1,55 \pm 0,002^{\mathrm{b}, \mathrm{c}} \\
1,57 \pm 0,003^{\mathrm{d}} \\
0,000\end{array}$ \\
\hline
\end{tabular}

DP: desarrollo puberal. $X_{ \pm} E E$ : Promedio \pm error estándar. EE: error estándar de la media. *Peso pronosticado en kg transformado mediante el inverso cuadrático. † Peso en kg normalizado por la raíz cuadrada. ₹ IMC en kg/mt2 transformado por el inverso cuadrático. § IMC en kg/mt2 normalizado por el inverso de la raíz cuadrada. IIANOVA de dos factores. Las letras repetidas en las columnas de peso, estatura e IMC por sexo denotan que no hubo diferencias significativas entre los pares $(p>0,05)$, aplicando la prueba post test de Bonferroni. 
individual, como se ilustrará con el siguiente ejemplo: de acuerdo con los datos obtenidos en los niños de Medellín, la diferencia de IMC entre un hombre en estadio $V$ de genitales externos y otro en estadio III fue de 2,6 unidades, que para un joven de 14,2 años equivale a 0,9 desviaciones estándar del IMC esperado para su edad y sexo; en consecuencia, si un hombre de dicha edad en lugar de estar en estadio III ya hubiera completado su desarrollo puberal, se ubicaría 0,9 desviaciones estándares por encima del valor que tendría si no hubiera completado su maduración, lo que llevaría a una clasificación nutricional errada.

Los valores de IMC, según desarrollo puberal, en los hombres de los colegios públicos de Medellín, fueron superiores a los reportados por otros dos estudios realizados en comunidades latinoamericanas de bajo nivel socioeconómico; uno en jóvenes de un establecimiento educativo público, del municipio Lima Blanco, área suburbana del Estado de Cojedes, en Venezuela, muestra tomada en 2007 (5), y el otro, en el complejo Macaco, localidad brasileña cuya muestra se recolectó en 1998 (20).

Las diferencias de IMC sugieren que las condiciones de vida de los niños de colegios públicos de Medellín son mejores que las de los jóvenes venezolanos y brasileños de los estudios mencionados. Al analizar la diferencia en IMC de los hombres que iniciaban su desarrollo puberal, con respecto a los que ya lo habían terminado, se observó que los de colegios públicos tuvieron una diferencia mayor a los de colegios privados $(3,5$ vs 2,8 unidades), situación que también se ha presentado en

\section{FIGURA 1}

Comportamiento del peso, la estatura y el IMC según estadio de desarrollo puberal y tipo de colegio por sexo.
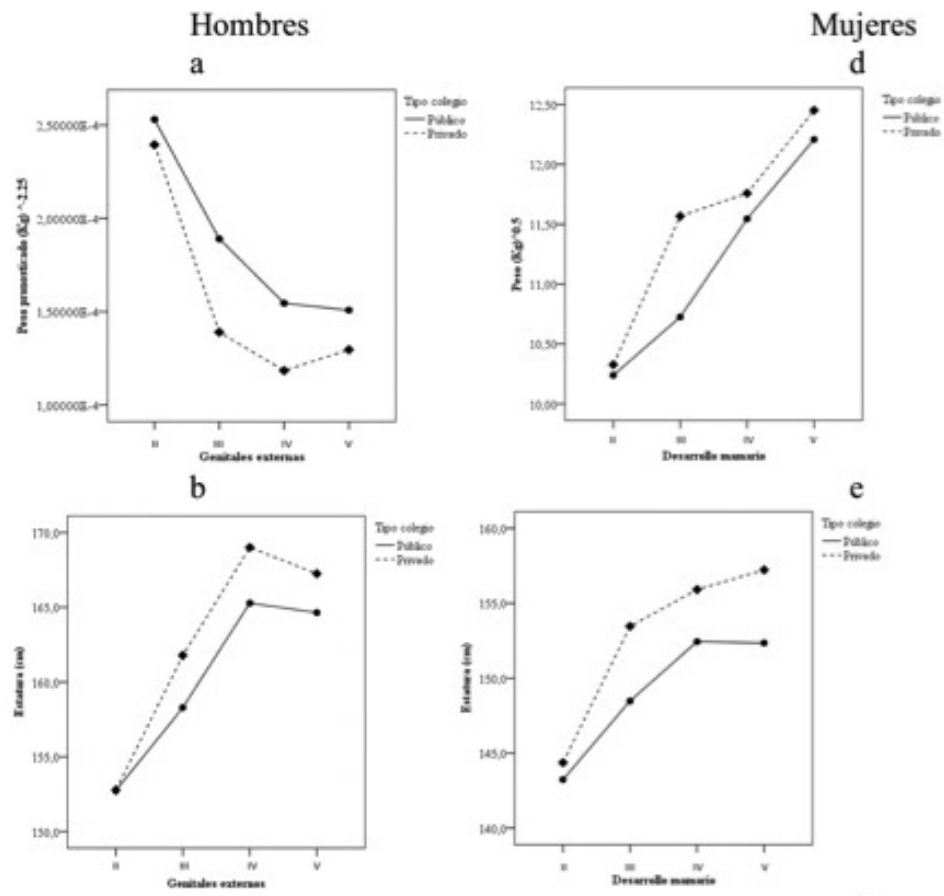

c
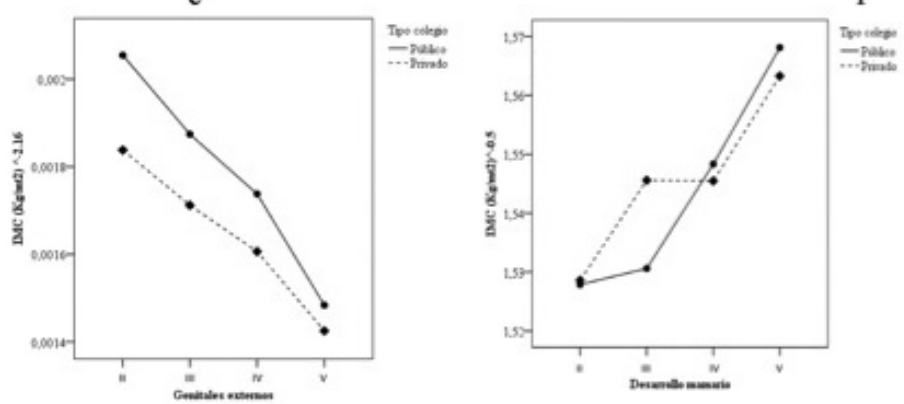

Las figuras $a, b$ y c corresponden al peso, estatura e IMC de los hombres respectivamente y las figuras d, e y $f$, al peso, estatura e IMC de las mujeres respectivamente. Las líneas continuas corresponden a los colegios públicos y las discontinuas a los privados.

Nota: las figuras a y c en hombres se presentan según el inverso cuadrático del valor real. 
otros estudios (23).

Los promedios de IMC en todos los estadios de desarrollo puberal de las mujeres de los colegios subvencionados de Chile fueron superiores a los que presentaban las mujeres de Medellín de ambos tipos de colegios; sin embargo, al inicio del desarrollo puberal las diferencias fueron mayores con las jóvenes de los colegios públicos y al final de la maduración con las de los colegios privados, sin duda como consecuencia del comportamiento del IMC según tipo de colegio en las mujeres de Medellín, puesto que en los estadios II y III de desarrollo puberal el IMC fue mayor en las mujeres de los colegios privados, pero la situación se invirtió en los estadios puberales IV y V, lo cual sugiere, en este grupo poblacional, la presencia de arquetipos asociados con la delgadez, documentados por Arboleda y colaboradores (24) en el estudio de cultura somática de los adolescentes de Medellín, realizado en la misma época en que se recogieron los datos de la investigación que suministró la muestra del presente estudio.

En la investigación de cultura somática, el motivo más frecuente de insatisfacción con el peso fue "el querer estar más delgado", tendencia más marcada en el grupo de 14 años, en mujeres y particularmente en aquellas de estratos más altos. El querer ser más delgada sin tener sobrepeso implica que el modelo estético asumido por las adolescentes corresponde al impuesto por la industria del modelaje, en la cual impera la delgadez (12). En niñas de 14 años se ha detectado una mayor preocupación por controlar su peso, como resultado de la influencia sociocultural, debido a que asocian el ser ricas, populares y exitosas con el hecho de ser bellas y lo que implica no tener exceso de peso (25).

Adicionalmente, el comportamiento del IMC en la muestra de mujeres de Medellín al finalizar el desarrollo puberal (estadio V), es similar al reportado en países desarrollados, donde las mujeres de condiciones socioeconómicas menos favorables presentaron promedios de IMC mayores, situación explicada por la mayor disponibilidad de alimentos de alta densidad energética, de gran tamaño y bajo costo, acompañada de un estilo de vida sedentario $(26,27)$.

Al comparar los promedios de IMC de las mujeres que iniciaban la pubertad con respecto a las que se encontraban en estadio $V$, se observó que las de los colegios públicos presentaron mayores diferencias, situación que muestra el mayor riesgo que tienen las adolescentes de condiciones socioeconómicas menos favorables de presentar sobrepeso u obesidad en su vida futura, adicionalmente se observó una diferencia mayor en las mujeres que en los hombres; esto también fue reportado por King (23).

En conclusión, los resultados de esta investigación corroboran que las condiciones socioeconómicas adversas limitan la posibilidad de crecimiento en peso y talla de los adolescentes, impidiendo que alcancen su máximo potencial. Igualmente, puso en evidencia que en las mujeres el efecto de las condiciones socioeconómicas sobre el IMC está mediado por otros factores, posiblemente culturales, que ponen a las mujeres de los colegios privados en mayor riesgo de desarrollar déficit de peso y a las de los colegios públicos mayor riesgo de exceso.

Las diferencias en IMC según desarrollo puberal son claras en adolescentes de ambos sexos de Medellín, y deberían tenerse en cuenta en la evaluación individual del estado nutricional por este indicador.

\section{RESUMEN}

Introducción: La ciudad de Medellín carece de investigaciones sobre variaciones del crecimiento según grado de desa- rrollo puberal y condiciones socioeconómicas en adolescentes. Objetivo: analizar la variabilidad de peso, estatura e índice de masa corporal (IMC) según grado de desarrollo puberal y tipo de colegio en adolescentes de Medellín. Sujetos y métodos: de la investigación "Factores de riesgo cardiovascular de Medellín en 1998-1999", se seleccionaron adolescentes de ambos sexos en estadios II al V de desarrollo puberal según Tanner (genitales externos en hombres y desarrollo mamario en mujeres). Se analizaron los datos de peso, estatura e IMC ( $\mathrm{kg} / \mathrm{m} 2)$, según desarrollo puberal y tipo de colegio público o privado. Resultados: los varones de los colegios privados tuvieron un peso $(p=0,000)$, estatura $(p=0,001)$ e IMC $(p=0,002)$ superiores a los de los públicos, sin interacción del desarrollo puberal y tipo de colegio $(p>0,05)$. En las mujeres, el peso y la estatura fueron superiores en las de colegios privados $(p=0,000)$ con interacción entre desarrollo puberal y el tipo de colegio para ambos ( $p=0,013$ y 0,040 respectivamente); el tipo de colegio no influyó sobre el IMC ( $p=0,347)$, pero si hubo interacción con el desarrollo puberal $(p=0,013)$, al inicio el IMC fue mayor en las de colegios privados y la situación se invirtió en las de estadio $\mathrm{V}$ de desarrollo mamario. Conclusión: el grado de desarrollo puberal influye en el crecimiento de varones y mujeres; sin embargo la magnitud de la diferencia en IMC y los estadios en que ocurren podrían estar relacionados con factores culturales en las mujeres.

Palabras clave: adolescente, peso corporal, estatura, índice de masa corporal, grado de desarrollo puberal, tipo de colegio.

Agradecimientos: Los autores agradecen a los investigadores del estudio de factores de riesgo cardiovascular en la población escolar y adolescente de Medellín: Ligia Martínez M., Martha Cecilia Álvarez U., Ivonne Laguado S., Wilmer Soler T., Rose Mery Arias, Jesús A. Camacho P., Jaime A. Pérez G. y a Juan Carlos Aristizábal R., por facilitar la base de datos para el presente estudio. Igualmente agradecen a la Estrategia de Sostenibilidad 2012-2013, de la Vicerrectoría de Investigación de la Universidad de Antioquia y al Grupo de Investigación en Alimentación y Nutrición Humana, Escuela de Nutrición y Dietética, Universidad de Antioquia, por su apoyo financiero para el desarrollo de la investigación.

\section{BIBLIOGRAFÍA}

1. Restrepo MT. Estado nutricional y crecimiento físico. 1a ed. Editorial Universidad de Antioquia. Medellín; 2000.

2. Mansilla G. Maduración biológica en la adolescencia. Rev Soc Bol Pediatr 2000;39:11-5.

3. Mauricci A, Burrows $R$, Leiva L, Zvaighaft A, Kauschansky A, S. M. Evaluación de la estatura durante el desarrollo puberal. Rev Chil Pediatr 1986;57:497-501.

4. Burrows R, Díaz M, Muzzo S. Variaciones del índice de masa corporal (IMC) de acuerdo al grado de desarrollo puberal alcanzado. Rev Med Chile 2004;132:1363-8.

5. Guerrero A, Sánchez $A$. Índice de Masa Corporal según grado de desarrollo puberal en varones venezolanos. An Venez Nutr 2009;22:20-4.

6. Comisión sobre Determinantes Sociales de la Salud. Subsanar las desigualdades en una generación. Alcanzar la equidad sanitaria actuando sobre los determinantes sociales de la salud: OMS; [Internet]. OMS; 2008. [Fecha de consulta: 10 de septiembre de 2009]. Disponible en: www.who.int/ social_determinants.

7. Webb EA, Kuh D, Pajak A, Kubinova R, Malyutina S, Bobak M. Estimation of secular trends in adult height, and 
childhood socioeconomic circumstances in three Eastern European populations. Econ Hum Biol. 2008;6:228-36.

8. Cardoso HF, Caninas M. Secular trends in social class differences of height, weight and $B M /$ of boys from two schools in Lisbon, Portugal (1910-2000). Econ Hum Biol 2010;8:111-20.

9. Rosique J, Restrepo MT. Asociación de la morfología corporal con el estrato socioeconómico en adolescentes de Medellín (Colombia). Rev Esp Antrop Fís 2003;24:49-62.

10. Hernández MI, Unanue N, Gaete X, Cassorla F, Codner E. Age of menarche and its relationship with body mass index and socioeconomic status. Rev Med Chil 2007;135:142936.

11. Restrepo MT, Álvarez MC, Quintero D, Londoño JL. Evaluación del estado nutricional de los escolares de Medellín: 1982 - 1983. Editorial Universidad de Antioquia, Medellín; 1983.

12. Restrepo MT, Aristizábal JC, Camacho JA, Velázquez MC. Cultura somática de adolescentes de 14-17 años escolarizados en Medellín: Características antropométricas. Perspect Nutr Human 2002;8:50-65.

13. Uscátegui RM, Álvarez MC, Laguado I, Soler W, Martínez $L$, Arias $R$, et al. Factores de riesgo cardiovascular en niños de 6 a 18 años de Medellín (Colombia). An Pediatr 2003;58:411-7.

14. Lohman TG. Anthropometry and body composition. In: Anthropometric standardization reference manual.1th ed. Champion: Human Kinetics; 1988, p. 1-130.

15. Marshall WA, Tanner JM. Variations in pattern of pubertal changes in girls. Arch Dis Child 1969;44:291-303.

16. Marshall WA, Tanner JM. Variations in the pattern of pubertal changes in boys. Arch Dis Child. 1970;45:13-23.

17. Landaeta-Jiménez M, Pérez BM, Escalante Y. Adiposidady patrón de grasa en jóvenes venezolanos por estrato social. Arch Latinoam Nutr 2002;52:128-36.

18. Barros AJ, Victora CG, Horta BL, Goncalves HD, Lima RC, Lynch J. Effects of socioeconomic change from birth to early adulthood on height and overweight. Int J Epidemiol
2006:35:1233-8.

19. Burrows A, Ceballos S, Burgueno M, Muzzo B. Trends in puberal development of school age children living in the Metropolitan Region of Chile. Rev Med Chil 2010;138:617.

20. Coehlo $K$, Sichieri $R$, González C. Influencia de la maduración sexual en el índice de masa corporal en adolescentes de bajo nivel socioeconómico de Rio de Janeiro. Rev Chil Nutr. [Revista en Internet] 2002; 29. [Fecha de consulta: 1 de junio de 2010]. Disponible en: http:// www.scielo.cl/scielo.php?script=sci_arttext\&pid=S071775182002000100005\&lng=pt\&nrm=iso\&tlng=es.

21. World Health Organization. Growth reference data for 5-19 years. WHO; 2007. [Fecha de consulta: 4 de junio de 2010]. Disponible en: http://www.who.int/growthref /en/.

22. Colombia. Ministerio de Protección Social. Resolución 2121. Junio de 2010. [acceso 4 de junio de 2010]. Disponible en línea: http://www.mapadehambre.com/resolucion-2121oms.pdf.

23. King T, Kavanagh AM, Jolley D, Turrell G, Crawford D. Weight and place: a multilevel cross-sectional survey of area-level social disadvantage and overweight/obesity in Australia. Int J Obes 2006;30:281-7.

24. Arboleda $R$, Duque B, Díaz B, Correa E, Castro J, López $A$, et al. El cuerpo en boca de los adolescentes. Estudio interdisciplinario de la cultura corporal en adolescentes de la ciudad de Medellín. Medellín: Kinesis; 2002.

25. Manrique RD, López PL, Cárdenas N, Toro E. Factores predisponentes de la anorexia y bulimia en el contexto de las universidades. Tesis de grado. Universidad CES. 2007.

26. O'Dea JA, Nguyen Hoang TD, MJ. D. Plateau in obesity and overweight in a cross sectional study of low, middle and high socioeconomic status schoolchildren between 2004 and 2009. Int J Public Health 2011;56:663-7.

27. Garnier D, Simondon KB, Hoarau T, Benefice E. Impact of the health and living conditions of migrant and non-migrant Senegalese adolescent girls on their nutritional status and growth. Public Health Nutr 2003;6:535-47. 